\title{
Un estudio psicoanalítico sobre la afirmación de lo simbólico por el sujeto
}

\section{A psychoanalytic study about the affirmation of the symbolic by the subject}

\section{Lucía Mauro}

Correspondencia:

luciamauro85@hotmail.com
Filiaciones Institucionales:

Instituto Universitario Italiano de Rosario IUNIR (Argentina)

Resumen: La tesis de Maestría titulada "La asunción de lo simbólico por el sujeto. Incidencias subjetivas y clínicas de esta operación fundante y sus posibles extravíos" responde a la pregunta por el origen de lo simbólico y sus consecuencias para la estructuración subjetiva.

Se realiza entonces un estudio exhaustivo de las operaciones de constitución del sujeto al tiempo que se problematiza su valor clínico para la diferenciación diagnóstica (Bejahung-Ausstossung y Verwerfung).

La tesis rastrea dichas operaciones para historizar su emergencia en el corpus psicoanalítico y analizar tanto su construcción teórica como su devenir a lo largo de la enseñanza de Freud y Lacan.

Siguiendo otra línea de investigación, se da tratamiento a lo simbólico afirmado por el sujeto en su articulación con los registros real e imaginario.

Como resultado, la tesis arriba a un cuestionamiento del valor operatorio de tales constructos conceptuales para dirimir el asunto psicosis-neurosis que interpela, de la misma manera, la clásica nosografía freudiana.

Palabras Clave: Bejahung - Simbólico - Psicoanálisis - Verwerfung - Ausstossung

Cómo citar:

Mauro, L Un estudio psicoanalítico sobre la afirmación de lo simbólico por el sujeto en Revista Psicoanálisis en la Universidad $\mathrm{N}^{\circ} 4$. Rosario, Argentina, UNR Editora.

Pág 167-179
ISSN: 2683-9938 (en línea)

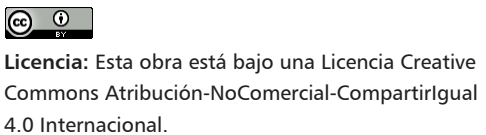

Responsabilidad editorial:

Universidad Nacional de Rosario.

Argentina. Facultad de Psicología.
Recibido:

$22-04-2019$

Aceptado:

$11-06-2020$

Publicado:

$05-10-2020$ 



\section{DE UNA CUESTIÓN PRELIMINAR A} TODA ESCRITURA POSIBLE DE UNA TESIS SOBRE LA bejabung

La tesis de Maestría "La asunción de lo simbólico por el sujeto. Incidencias subjetivas y clínicas de esta operación fundante y sus posibles extravíos» es el precipitado de un interés que comienza ya en el tiempo del pregrado y que tomó verdadera consistencia durante el cursado de la Maestría en Psicoanálisis, dictada en la Facultad de Psicología de la Universidad Nacional de Rosario.

En consecuencia, la escritura de dicha tesis recoge las marcas del pasaje por la cátedra de Clínica II A, cuyo programa, para el año 2008, abordaba, como punto de una de sus unidades, la cuestión de la Bejahung en su articulación con la locura.

Es así como, una inquietud incipiente por el asunto del origen fue tomando el estatuto de una investigación a causa de la importancia tanto teórica como clínica que la asunción de lo simbólico ha tenido para el psicoanálisis.

Ya presente en los escritos freudianos, la referencia a este acto originario se encuentra, una y otra vez, en diferentes momentos de la enseñanza de Lacan. La insistencia de este tema en el corpus teórico psicoanalítico resulta entonces irrefutable.

Desde referencias fragmentarias y puntuales hasta escritos que hacen de ella el eje central, la Bejahung escande la doctrina de Lacan y, allí donde no se la nombra explícitamente, no deja por ello de ser evocada de manera alusiva. Cabe advertir, sin embargo, una cierta rebaja en el tratamiento dado por Lacan a la Bejahung paralelamente a la avanzada creciente de las cuerdas y los nudos. Rebaja que, lejos de restar importancia a la afirmación de lo simbólico, indica, antes bien, un tiempo de invención de lo real que permita nuevas articulaciones del registro simbólico mismo.

La tesis señala la relevancia del tema de la afirmación de lo simbólico en tanto se trata de un operador teórico que permite dirimir lo preliminar a todo tratamiento posible, es decir, la división estructural psicosis/neurosis.

Asimismo, el desarrollo argumentativo interroga la fecundidad de dicho criterio diferencial para dar cuenta, de manera acabada, de esa bifurcación subjetiva. De acuerdo con esto, en un primer tiempo de la investigación, se buscó precisar cuáles son las huellas de la Bejabung en la economía subjetiva para luego analizar si las mismas resultan suficientes al momento de determinar neurosis o psicosis. Siguiendo esta línea de exploración es que se propuso una serie de fenómenos clínicos para ser interrogados desde la matriz de la Bejahung y que van desde la negación discursiva hasta el negativismo delirante, pasando por un espectro intermedio que engloba las anorexias, bulimias, toxicomanías y los fenómenos de extrañeza.

Ese apuntalamiento clínico retornó sobre la teoría introduciendo modulaciones sobre la hipótesis fundamental, ofrecida por Lacan en el seminario Las Psicosis: "En el origen hay pues Bejahung, a saber, afirmación de lo que es, o Verwerfung" (Lacan, 1955/1956, p. 120).

La tesis parte de este binarismo y se propone indagar las consecuencias subjetivas o, más bien, subjetivantes del mismo al tiempo que interpela su formulación en términos dicotómicos y opositivos. En este sentido, la tesis da tratamiento entonces a los accidentes en el seno de la Bejahung y trabaja, pieza por pieza, las referencias del seminario Las Psicosis para señalar las 
variaciones de la enunciación en el enunciado de Lacan.

Es así como nos topamos con que, por una parte, la ausencia de Bejahung convive con la ausencia en la Bejahung, es decir, con la Verwerfung en la Bejabung y, por otra parte, la Verwerfung se apelmaza asintóticamente con otros mecanismos, fundamentalmente con el de Ausstossung. Todo el desarrollo de la tesis gira alrededor de estas problemáticas.

Se realiza un estudio exhaustivo de los mecanismos estructurantes del sujeto que resalta la complejidad de esa operación de constitución. Para ello, la tesis desarrolla un procedimiento metodológico argumentativo de modalidad ensayística en torno a las cuestiones fundamentales a los fines de recoger su carácter problemático sin ahogarlas en una construcción totalizadora y sistemática. El ensayo incurre en una crítica de los conceptos presentados en su interacción dejando de lado definiciones estrictas e hipostasiadas (Adorno, 1962). En este sentido, dicho género responde de manera privilegiada al interés del tema que impone considerar la equivocidad arrastrada por cada una de las operaciones con que se ha pretendido nombrar el mecanismo fundante del sujeto.

La tesis consta de una introducción que, a modo de cuestión preliminar, aproxima al tema arrojando algunos tópicos que serán desarrollados en los capítulos. El cuerpo de la tesis se divide en ocho capítulos que avanzan partiendo de un tratamiento pormenorizado del tema alrededor de las cuestiones principales: articulación Bejahung-Ausstossung y Verwerfung, destinos de la Bejahung (Verneinung, Verdichtung, Verdrängung) y retornos de la Verwerfung para, luego, pasar a profun- dizar acerca de lo simbólico afirmado por esa Bejahung.

Atendiendo a este último asunto, se busca establecer la relación entre la afirmación de lo simbólico y las operaciones de metáfora y metonimia al tiempo que se indaga un posible abordaje topológico de la misma.

En este punto del recorrido, eminentemente teórico, la tesis explora el nexo de la afirmación simbólica con el álgebra de Lacan, sus matemas y las cuerdas a los fines de interrogar su transmisión en la enseñanza de aquel.

A continuación, la investigación pasa a indagar los "antecedentes" de dicha operación en Ferenczi, al recuperar sus teorizaciones acerca del mecanismo de introyección en tanto se busca precisar qué clase de operación es esa admisión de lo simbólico.

El abordaje teórico de la afirmación simbólica también contempla sus incidencias sobre la sexuación interrogando entonces el producto de esa afirmación.

Finalmente, en el último tramo, la tesis pasa a realizar un abordaje clínico de la Bejahung tal como se nos revela en el dispositivo analítico. Así, el trabajo se centra, en profundidad, en correlacionar los efectos subjetivos de la admisión de lo simbólico en el analizante (abarcando desde la negación discursiva hasta el negativismo no negador e incluyendo también la fallida incorporación-expulsión anoréxica y la extrañeza de un cuerpo imaginario producto de las sustancias "tóxicas") con las intervenciones posibles del analista (desde la construcción/interpretación hasta la posición de secretario). 
INTRODUCCIÓN AL COMENTARIO DE LACAN SOBRE La bejahung DE FreUd

Ahora bien, la tesis, en su aspecto teórico, se centra fundamentalmente en el tratamiento dado a la Bejahung por Lacan en el seminario Las Psicosis. Si bien hay referencias a la misma anteriores a este seminario, por ejemplo en el Seminario Los Escritos Técnicos de Freud (1953/1954), será aquel en que la asunción de lo simbólico reciba una precisión conceptual inédita hasta entonces. En este sentido, cabe esperarse que la Bejahung, como un operador de lectura que viene a responder a la pregunta por el origen de lo simbólico en el sujeto, sea ajustada teóricamente en la época hipersimbolista de Lacan. Es por esta razón entonces que la tesis tiene su zócalo en el seminario del 55/56 y en "De una cuestión preliminar a todo tratamiento posible de la psicosis" (Lacan, 1957/1958).

Sin embargo, esta Bejahung tiene su antecedente en Freud. La Bejahung, entendida como afirmación, es introducida por Freud en su texto "La negación" (1925). Allí, es presentada como una de las dos decisiones que cabe atribuir a la función del juicio y en correspondencia con ese yo-placer-originario, ya mencionado en "Pulsiones y destinos de pulsión" (Freud, 1915).

La Bejahung, en la letra freudiana, está lejos de ser una operación, queda confinada a una función del juicio consistente en atribuir o desatribuir una propiedad a una cosa y es condición de posibilidad del juicio de existencia por el cual se admite o se impugna la existencia de una representación en la realidad. Ya allí es posible situar la paradoja de atribuir una propiedad a algo que pudiere ser inexistente pero paradoja efectiva en tanto insinúa, preci- samente, que la existencia ha de ser esperada del símbolo. Es el símbolo atribuido (la propiedad) lo que confiere la existencia o inexistencia a la cosa.

$\mathrm{La}$ introducción freudiana de la $\mathrm{Be}$ jahung es equívoca y de estofa pulsional: "Quiero comer o quiero escupir esto (...) Quiero introducir esto en mí o quiero excluir esto de mí (...) introyectarse todo lo bueno, arrojar de sí todo lo malo" (Freud, 1925, p. 254). Cabe detenerse entonces en el carácter heteróclito de los términos: comer, introducir, introyectar, por un lado, y excluir, arrojar, por otro; a los que se agregan recoger e incorporar y expeler y proyectar, hallados en "Pulsiones y destinos de pulsión” (Freud, 1915).

Lacan despeja la equivocidad en lo que refiere a la Bejahung a partir de su ternario R.S.I mientras que su reverso negativo, Ausstossung, arrastrará consigo esa equivocidad aproximándose a otra forma de exclusión que Lacan construye, en el seminario III, y que llama Verwerfung.

Para Lacan, la Bejahung es la afirmación o admisión de un primer cuerpo de significantes y se opone a la Verwerfung: "En el origen hay pues Bejahung, a saber, afirmación de lo que es, o Verwerfung" (Lacan, 1955/1956, p. 120).

Respecto de la Verwerfung, Lacan afirma que "Se trata del rechazo, de la expulsión, de un significante primordial a las tinieblas exteriores, significante que a partir de entonces faltará en ese nivel" (Lacan, $1955 / 1956$, p. 217) y la ubica como mecanismo de base de la paranoia.

De acuerdo con esto, si hay Verwerfung, no hay Bejahung. La Verwerfung toma el valor de una ausencia de $B e$ jabung de modo tal que la oposición Bejahung-Verwerfung deviene un criterio de diferenciación entre neurosis y psico- 
sis. Lacan lo explicita indicando que "en el fenómeno de la psicosis: es el término Verwerfung. Se articula en ese registro como la ausencia de esa Bejahung" (Lacan, 1957/1958, p. 534).

Tenemos así tres operaciones: por un lado, una operación de afirmación llamada Bejahung y, por otro lado, dos operaciones de exclusión: Ausstossung y Verwerfung.

La tesis se detiene en un análisis detallado de los mecanismos de constitución del sujeto a los fines de introducir las diferencias que los separan pero también indicar la fragilidad e insuficiencia de esas mismas distinciones. En consecuencia, el desarrollo argumentativo se centra en un examen minucioso de la operación de afirmación a la luz de una serie de interrogantes que van desde el objeto afirmado hasta el sujeto de esa afirmación sin olvidar la especificidad de sus coordenadas espacio-temporales y, menos aún, el desnivel entre la introducción freudiana del juicio de la Bejahung y la construcción lacaniana de la operación de la Bejahung.

De modo que la exposición se sostiene en un trabajo a la letra que va recuperando las inconsistencias y vacilaciones, las temblorosas distinciones, los desplazamientos de la significación y las sutiles sustituciones con que el asunto Bejahung-Ausstossung ha sido abordado a lo largo de la enseñanza de Lacan, esquivando cualquier tentativa de tratamiento imaginario de la cuestión.

La tesis parte, en su aproximación a la Bejahung, de una suposición de Lacan, de una proposición dogmática: "Es estructuralmente necesario postular una etapa primitiva en la cual aparecen en el mundo significantes en cuanto tales" (Lacan, 1955/1956, p. 215). Aparición ésta que ya implica el lenguaje, aclara Lacan, y que es enunciada como su tesis. Entonces, la tesis parte de la tesis de Lacan, "la realidad está marcada de entrada por el anonadamiento simbólico” (Lacan, 1955/1956, p. 214).

Ahora bien, esta postulación entraría en contradicción con la proposición de una Bejahung (que justamente supone al símbolo como no dado de antemano sino como el producto de un acto de afirmación) si se recubrieran imaginariamente el problema de lo simbólico en el mundo con el de lo simbólico en el sujeto. Lo que aparece en el mundo no aparece, por ello, en el sujeto pues el hay, de los significantes en lo real, no obedece a la economía de la Bejabung y, por lo tanto, no puede faltar mientras que hay un simbólico en el sujeto que no se basta a sí mismo y que debe su aparición al acto de afirmación.

Sin embargo, entre lo simbólico en el mundo y lo simbólico en el sujeto hay una articulación pues los significantes en lo real tanto más nos hablan cuanto menos hablamos.

Así, el enfoque dado a la Bejabung en la tesis reconquista las diferencias ahí donde amenaza un punto de confusión y de apelmazamiento teórico pero no sin trazar, a su vez, la articulación entre eso mismo diferenciado.

La precisión en la distinción entre un simbólico en el mundo y un simbólico en el sujeto advierte de no imaginarizar lo simbólico (que así se revela como no siendo ni uno ni el mismo) y de no hacer equivaler ausencia de Bejahung a ausencia de simbólico. Por lo tanto, se plantea que hay un simbólico que ya no responde a la legalidad de esa admisión.

Atendiendo a este punto es que, a continuación, la tesis se centra en un análisis intensivo del objeto de la Bejahung, es de- 
cir, de ese simbólico afirmado por el sujeto.

Desde una matriz de signos de percepción (Wahrnehmungszeichen) a una parte del texto simbólico pasando por un cuerpo de significantes, el simbólico afirmado vuelve a dividirse, sigue sin ser uno.

De la misma manera, la fractura del objeto de la Bejahung se reintroduce por la vía del antecedente freudiano. La Bejahung de "La negación” (1925) recae sobre "lo bueno", "lo placentero" o "una propiedad" (de la cosa) revelando también que esa función del juicio (que es la $B e$ jabung en manos de Freud) ya supone la introducción del símbolo como condición de posibilidad de enjuiciar, ya cuenta con el símbolo que la Bejahung lacaniana haría ingresar.

En consecuencia, se propone pensar que la Bejabung freudiana responde a la pregunta por el comienzo (Anfang) antes que por el origen (Ursprung), como sí la encontramos desarrollada por Lacan. Es Freud quien la lee con clave yoica y la construye como una afirmación que toca a un yo-placer-originario cuya "originariedad" queda al mismo tiempo desmentida por el carácter primario con que Freud la predica.

La investigación continúa por el interrogante acerca de quién afirma y quién se afirma en y por ese acto.

Mientras Lacan señala que "no basta con que el sujeto haya elegido en el texto de lo que hay que decir, una parte (...) para que al menos con esa las cosas encajen bien" (Lacan, 1955/1956, p. 120) introduciendo el problema de un sujeto que antecede al acto mismo de elección que lo constituiría como tal. Freud resuelve la aparente contradicción con el dualismo yoico que separa un yo-placer-originario de un yo-realidad-definitivo.
Cuestionando la idea de un sujeto como uno e idéntico a sí mismo (la yoización de un sujeto) es que se propone resolver la falsa antinomia planteando que no hay identidad entre el sujeto que afirma, productor de esa afirmación, y el sujeto afirmado, producto de la misma.

Sin profundizar en la multiplicidad de sentidos que el concepto de sujeto tiene en la teoría de Lacan, la tesis busca interpelar ciertas lecturas simplistas que han pretendido zanjar la cuestión del sujeto en la psicosis por la vía de un binarismo entre hay sujeto o no hay sujeto. Tales planteos han abrochado el "hay Bejahung" con "hay sujeto" y el "no hay Bejahung" (atribuido a la psicosis) con un "no hay sujeto".

Tomando una posición alternativa, se plantea la pregunta por el sujeto del que se trata en la psicosis ahí donde no hubo acto (o, hubo un no-acto) que lo constituya en cuanto tal.

En el seminario Las Psicosis, Lacan no vacila en hablar de "sujeto psicótico" pues, en ese contexto, el sujeto aparece definido como tal por el sólo hecho de hablar: "Están en presencia de un sujeto en la medida en que lo que dice y hace -es lo mismo- puede suponerse haber sido dicho y hecho para engañarlos" (Lacan, 1955/1956, p. 58). Asimismo, en “De una cuestión preliminar a todo tratamiento posible de la psicosis" (1957/1958), nos encontramos también con referencias a un sujeto en la psicosis pero aquí ya no se trata de un sujeto con intención de engaño (por la vía del significante) sino de un sujeto con intención de rechazo (del objeto), como ocurre en la alucinación.

Así es como el discurso acabó por realizar su intención de rechazo en la alucinación. En el lugar donde el objeto indecible es rechazado en lo real, se deja oír una 
palabra, por el hecho de que, ocupando el lugar de lo que no tiene nombre, no ha podido seguir la intención del sujeto. (Lacan, 1957/1958, p. 513)

En consecuencia, respecto de la cuestión del sujeto en la psicosis tampoco es posible decir que se trate del mismo sujeto.

Ahora bien, la tesis vuelve, una $\mathrm{y}$ otra vez, sobre la afirmación de Lacan: hay Bejahung o hay Verwerfung (Lacan, $1955 / 1956$, p. 120). Para poder avanzar, la exposición tiene que volver a ese punto en tanto resulta una afirmación problemática, con múltiples implicancias. Otra arista que se desprende de esta aseveración lleva a interrogar el reverso negativo de la Bejahung, la faz expulsiva de ésta, introducida por Freud en "La negación” (1925) como Ausstossung. Conjuntamente con la Bejahung, esta expulsión se opone a la Verwerfung.

Sin embargo, el acercamiento profundo a la operatoria de la Ausstossung conduce a relativizar tal oposición al punto de resaltar su temida cercanía a la forclusión. Siguiendo esta línea, la tesis ensaya posibles vías por las cuales rehallar la diferencia entre Ausstossung y Verwerfung.

Así es que se imponen tres vertientes, la primera concierne a la diversidad del rincón freudiano por donde asoman ambas operatorias. Si la Ausstossung emerge en "La negación", la Verwerfung es construida por Lacan a partir de la alucinación del hombre de los lobos.

La segunda vertiente es la vía imaginaria del sentido que también las bifurca puesto que la Ausstossung es significada como expulsión mientras que la Verwerfung toma la valencia de un rechazo.

La tercera es la vía del objeto sobre el cual recaen y que opone la Ausstossung de lo real a la Verwerfung del significante (de lo simbólico).

El desarrollo espiralado de la tesis permite que una vez trazadas esas distinciones se vuelva sobre ellas para indicar el punto de fracaso en la tentativa de diferenciación pero sin que ello lleve a pronunciarse por la no-diferenciación.

Basta recordar la "Respuesta al comentario de Jean Hyppolite sobre la Verneinung de Freud" (1956) para ver cómo Lacan pone en continuidad Ausstossung y Verwerfung. En este escrito, las trata indistintamente y ahí donde introduce la Verwerfung termina hablando de la Ausstossung cuestionando, de esta manera, su valor operatorio para una diferenciación diagnóstica entre psicosis $\mathrm{y}$ neurosis.

Asimismo, la distinción por la vía de la significación resulta poco diferenciadora puesto que basta citar al mismo Lacan, en el seminario III, para que "rechazo" y “expulsión” queden situados en una serie metonímica moebiana sin corte. "Se trata del rechazo, de la expulsión, de un significante primordial" (Lacan, 1955/1956, p. 217). En la misma dirección y tomando "La negación" de Freud, Lacan afirma que "Hay en la dialéctica de Freud una primera división de lo bueno y lo malo que sólo puede concebirse si la interpretamos como el rechazo de un significante primordial" (Lacan, 1955/1956, p. 218). La Verwerfung es leída en el lugar mismo de la operatoria de la Ausstossung y será a la Verneinung primordial a la que le de la primera significación significante de la Verwerfung. (Lacan, 1959/1960, p. 82)

Y la tercera vía, la del objeto sobre el que recaen, sufre idéntico destino puesto que la repartición simple, Ausstossung de 
lo real y Verwerfung de lo simbólico, se desbarata cuando nos topamos, al mismo tiempo, con una Verwerfung de la Cosa y una Verwerfung del sujeto por el discurso de la ciencia (Lacan, 1959/1960), una Verwerfung originaria del ser (Lacan, 1966/1967) y una forclusión del sentido por la orientación de lo real (Lacan, 1975/1976).

Esta parte de la tesis examina minuciosamente tales mecanismos para culminar en la pregunta por dónde leemos la operatoria de un mecanismo y por la distinción entre estructuras.

El último ensayo de hallar la separación entre Ausstossung y Verwerfung, que resulte más consistente, recae sobre la modalidad de retorno de tales operaciones. Sin embargo, el retorno en lo real, privativo del mecanismo de la forclusión que está en la base de la paranoia, toma su modelo de la alucinación del hombre de los lobos, cuya "historia de una neurosis infantil" ya introduce un punto de sospecha, al tiempo que Lacan hará equivaler la alucinación al acting out en "Respuesta al comentario de Jean Hyppolite sobre la Verneinung de Freud" (1956). En este sentido, solo si se corre el punto de distinción es posible conservar la modalidad de retorno como criterio diferenciador y plantear un retorno en lo real alucinatorio (para la Verwerfung) y un retorno en lo real no alucinatorio (para la Ausstossung).

El tiempo de comprender de la tesis se precipita a concluir que el punto de distinción de los mecanismos, en que se sostiene la diferencia estructural, vuelve a ser reintroducido en una economía de indiferenciaciones que exige un nuevo corrimiento de ese mismo punto.

Esa carretera principal del escrito toma también, en su desarrollo, una serie de des- víos para analizar las múltiples implicancias que la operación de la Bejahung tiene para el sujeto.

De acuerdo con esto, se indaga si la sexuación es un asunto de la afirmación simbólica o si cabe atribuírselo a la metáfora paterna.

Antes bien, se precisa la anterioridad de la operación de la Bejahung como operación de ausencia de la madre no superponible entonces con la sustitución metafórica por el Nombre del Padre, tal como se desprende de la afirmación de Lacan: "la metáfora del Nombre del Padre, o sea a la metáfora que sustituye el lugar primeramente simbolizado por la operación de ausencia de la madre, por este Nombre" (Lacan, 1957/1958, p. 533)

Hay un desnivel entre Bejahung y metáfora paterna que sugiere que la simbolización no viene de esta última mientras se ve inscribirse la Verdichtung entre los destinos de la Bejahung.

La relación de la Bejahung con la sexuación tiene un efecto en cascada pues lleva a interrogar si el producto de tal operación es el sexualmente opaco sujeto o si se trata de la diferencia (con no menos opacidad) entre hombre y mujer. Asimismo, esta cuestión retorna sobre la problemática bipolaridad inicial (o Bejahung o Verwerfung) al proyectar sobre la $B e$ jahung el piso forclusivo de lo femenino.

La matriz de la afirmación simbólica es un cuerpo de significantes donde faltan algunas lettres, entre ellas la que pudiere responder por qué es una mujer. Primer indicio de la posible cohabitación Bejahung-Verwerfung aunque esta Verwerfung no toque allí al Nombre del Padre.

La investigación prosigue en una demostración del modo en que la froclusión se va desprendiendo progresivamente del 
campo de la psicosis y del Nombre del Padre dejando así de ser el operador de lectura restringido a la locura para entrar en diferentes relaciones con el zócalo neurótico, ya sea como Verwerfung de los mandamientos de la palabra (Lacan, 1955), Verwerfung del phallus (Lacan, 1958/1959), forclusión de la Cosa por el discurso de la ciencia (Lacan, 1959/1960), Verwerfung del ser (Lacan, 1966/1967), Verwerfung del decir y Verwerfung del amor en el discurso capitalista (Lacan, 1971/1972) o como forclusión del sentido por la orientación de lo real (Lacan, 1975/1976).

A medida que la Verwerfung amplía su campo operatorio pierde su privilegio como criterio diagnóstico, pierde su especificidad como mecanismo propio de la psicosis.

Asimismo, tales precisiones en la consideración de la Verwerfung resultan fundamentales en tanto permiten precisar, a su vez, a la Bejahung.

La construcción de la Verwerfung y las variaciones en su conceptualización inciden en la conceptualización misma de la Bejahung. Siguiendo esta perspectiva es que la tesis toma los desarrollos de otros analistas a los fines de ir determinando cada vez más la operación de afirmación de lo simbólico.

En este momento de la tesis, se destaca la interlocución de Lacan con Melanie Klein, en las sesiones del seminario I Los Escritos Técnicos de Freud (1953/1954), para evitar cualquier tentativa de imaginarización del doble movimiento $\mathrm{Be}$ jahung-Ausstossung que los situara en una reversibilidad opositiva y complementaria.

Si bien la rítmica kleiniana introyección/proyección/re-introyección amenaza con hacer de la Ausstossung la anulación de la Bejahung, las resguarda de un tratamiento degradante en manos de Anna Freud. El apoyo en Klein vale como despedida de la lectura imaginarizante de los post-freudianos.

La intervención de Jean Hyppolite sobre la Verneinung de Freud es doblemente agradecida por Lacan. El filósofo no solo rectifica la traducción de Verneinung por “denegación” (en lugar de "negación”) sino que reintroduce el desnivel que coloca a la Bejahung operando en un plano no coincidente con el nivel operatorio de la Verneinung.

El pasaje de la Bejahung por la matriz filosófica la expresa en un lenguaje heideggeriano que la resguarda de toda aprehensión metafísica. Esta versión de la afirmación se perfila en las siguientes palabras de Lacan: "no es otra cosa sino la condición primordial para que de lo real algo venga a ofrecerse a la revelación del ser, o, para emplear el lenguaje de Heidegger, sea dejado-ser" (Lacan, 1954, p. 372)

Francois Balmes realiza un estudio exhaustivo centrado en esta formulación filosófica de la asunción de lo simbólico (Balmes, 2002)

Por otra parte, la equivocidad de los términos con los que Freud nombra esa afirmación primordial (comer, introducir, introyectar, recoger, incorporar) resulta problematizada a partir de las teorizaciones de Ferenczi acerca del mecanismo de introyección (Ferenczi, 1982)

Por último, si con Ferenczi esa afirmación se vuelve un asunto introyectivo, con los planteos del filósofo Jean Luc Nancy resalta la dimensión corporal en juego tanto de la Bejahung como de su faz expulsiva, Ausstossung: "una rítmica fundamental del afecto (...) la pulsación de una incorporación y una excorporación, de 
un aceptar/rechazar o un tragar/escupir" (Nancy, 2007, p. 79)

\section{LA BEJAHUNG EN LA DIRECCIÓN DE LA CURA. INTERVENCIONES SOBRE LA TRANSFERENCIA}

Seguidamente, la tesis pasa a una aproximación clínica de las incidencias subjetivas de la Bejahung. La introyección y expulsión implicadas en la afirmación de lo simbólico conduce a indagar los avatares de la oralidad como formas sintomáticas de la incorporación y del rechazo.

Ese "quiero comer" o "quiero escupir", con que Freud traduce al lenguaje de la pulsión oral el juicio de atribución, hace sospechar que las anorexias y bulimias son un asunto de Bejahung.

En este sentido, la tesis propone entonces una lectura de las mismas a partir de este operador teórico pero ya no absorbido en la dicotomía simple "hay" o "no hay" afirmación sino desde un "hay" que recoja las diferentes modalidades de su acontecer accidentado pues "en el seno de la Bejahung, ocurren toda clase de accidentes" (Lacan, 1955/1956, p. 121)

Partiendo de lo enunciado por Lacan en Radiofonía (1970), "el cuerpo de lo simbólico (...) aísla el cuerpo tomado en sentido ingenuo (...) El primer cuerpo hace que el segundo ahí se incorpore" (Lacan, 1970 , p. 18), es que la tesis explora las anorexias, bulimias y toxicomanías en su relación con lo Unheimlich así como también el negativismo propio del delirio de negaciones.

Se propone pensar tanto la cadaverización anoréxica del cuerpo, la "corpsficación” del cuerpo real, como la extrañeza del cuerpo imaginario (especular) por la embriaguez narcótica como efectos de los avatares en esa incorporación del cuerpo de lo simbólico.

Respecto del negativismo delirante, la tesis opone una negación que no es, una negación funcionando por fuera de la matriz de la Bejabung, a la negación en la neurosis como huella de la afirmación de lo simbólico. Para ello, toma el negativismo de las hermanas Papin o del presidente Schreber en el momento inicial de su delirio en contraste con la negación de las histéricas Anna O. y Lucy.

Siguiendo esta misma línea de investigación, es que se plantea que al analista toca escuchar el sí del analizante, inyectar la Bejahung, cuando la enunciación del "no" de la Verneinung arañe el enunciado atribuyendo entonces al sujeto su saber afirmado como negado. Saber tanto más existente cuanto más rechazado resulta por su resistencia yoica.

\section{MÁS ALLÁ DEL "PRINCIPIO DE LA bejabung"}

Como resultado de este recorrido, la tesis sugiere entonces cierta estrechez de esa trinidad teórica Bejahung-Ausstossung y Verwerfung para recoger la distinción estructural. Se propone pensar la insuficiente distinción de tales operadores conceptuales como derivada de la igualmente insuficiente distinción entre neurosis y psicosis puesto que se ha demostrado cómo la neurosis es pescada desde los operadores de la locura así como también los mecanismos psicóticos parasitan el dominio neurótico. Se pudo argumentar que las múltiples valencias de la Verwerfung, entre las cuales cabe situar su semblante de Ausstossung, denotan la pertinencia de los mecanismos no represivos en la neurosis así como de una modalidad de retorno de igual modo no bien ajustada a la represión. 
La semejanza de la sintomatología prepsicótica con la neurótica (Lacan, 1955/1956, p. 273) denuncia también que la fecundidad relativa de los criterios de diferenciación diagnóstica se agota en las estructuras declaradas o desencadenadas mientras que cierto modo de cohabitación entre neurosis y psicosis se evidencia por el hecho de que los mecanismos locos tomados de la segunda hacen posible leer a la primera.

La tesis deja abiertas algunas líneas de interrogación para futuras investigaciones que profundicen la cuestión del carácter limitado de los criterios de distinción estructural a partir de considerar no sólo el operador de diferenciación sino también el estatuto de lo diferenciable mismo.

La tesis conduce entonces a preguntar cómo un criterio simbólico podría dar cuenta de una diferencia que tal vez deba ser atribuida a lo real.

Finalmente, queda planteada entonces la inquietud por la supervivencia de la nosografía freudiana y por la construcción y análisis de otros operadores de lectura que respondan a la pregunta por la estructuración del sujeto.

\section{BIBLIOGRAFÍA}

Adorno, T. (1962) "El ensayo como forma" en Pensamiento de los confines, Número 5, 1998, 247-259. Buenos Aires, Argentina. Fondo de cultura económica.

Balmes, F. (2002) Lo que Lacan dice del ser. Buenos Aires, Argentina. Amorrortu.
Freud, S. (1915) "Pulsiones y destinos de pulsión” en Obras Completas (Vol. 14, pp. 105-134). Buenos Aires, Argentina, Amorrortu.

(1924[1923]) "Neurosis y psicosis” en Obras Completas (Vol. 19, pp. 151-159). Buenos Aires, Argentina, Amorrortu.

(1924) "La pérdida de realidad en la neurosis y la psicosis" en Obras Completas (Vol. 19, pp. 189-197) Buenos Aires, Argentina, Amorrortu. (1925) "La negación” en Obras Completas (Vol. 19, pp. 249-257). Buenos Aires, Argentina, Amorrortu. Lacan, J. (1981) Los Escritos Técnicos de Freud. Lacan. El seminario. Buenos Aires, Argentina, Paidós.

(1984) Las Psicosis. Lacan. El seminario. Buenos Aires, Argentina, Paidós.

(2006) El sinthome. Lacan. El seminario. Buenos Aires, Argentina, Paidós.

(2002) "De una cuestión preliminar a todo tratamiento posible de la psicosis" en Escritos. (Vol. 2, pp. 509-557). Buenos Aires, Argentina. Siglo XXI.

(1971) "Respuesta al comentario de Jean Hyppolite sobre la Verneinung de Freud" en Escritos. (Vol. 1, pp.366-383). Buenos Aires, Argentina. Siglo XXI.

(1977) Psicoanálisis. Radiofonía \& Televisión. Barcelona, España. Anagrama.

Nancy, J. (2007). A la escucha. Buenos Aires, Argentina. Amorrortu.

Rabinovitch, S. (2000) Encerrados afuera. La preclusión, un concepto lacaniano. Barcelona, España. Del Serbal. 


\section{LuCía Mauro}

Psicóloga, egresada en la Universidad Nacional de Rosario donde culminó su Maestría en Psicoanálisis. Actualmente, además de su labor clínica, se desempeña como docente en la Facultad de Psicología del Hospital Italiano de Rosario (IUNIR), en las cátedras de Psicopatología y Psicología Clínica de la Salud I y como supervisora de las práctica pre-profesionales. Es autora de Locuras. 7 invitaciones delirantes para una folie a deux con la escribiente (Laborde Editor, 2016). 